\title{
Industria 4.0 en México, la administración inteligente en sistemas productivos
}

\section{Industry 4.0 in Mexico, intelligent management in productive systems}

PINTOR-CORONA, Maura†*, GONZALEZ-MONZON, Ana Lilia y MENDOZA-SANTIAGO, Esther Tecnológico de Estudios Superiores de Jilotepec

ID $1^{\text {er }}$ Autor: Maura, Pintor-Corona

ID $2^{\text {do }}$ Autor: Ana Lilia, Gonzalez-Monzon

ID $3^{\text {er }}$ Autor: Esther, Mendoza-Santiago

DOI: $10.35429 / J I T C .2019 .8 .3 .25 .27$

Recibido 06 de Abril, 2019; Aceptado 25 de Junio, 2019

\section{Resumen}

Un modelo de negocio disruptivo es aquel esquema que ha cambiado de acuerdo con las nuevas tendencias de consumo de los clientes, convirtiendo a una industria de producción física a una forma de producción electrónica, abriendo nuevas oportunidades para la industria en México y convirtiéndose en un elemento transformador económico y social que ha permitido cambiar las reglas del juego, mediante implementación de desarrollos tecnológicos, que están creando un impacto en la forma de hacer negocios. A través de tecnologías como la Inteligencia artificial (con máquinas capaces de aprender), Robótica (potenciando la automatización), Internet de las cosas (con un mundo interconectado), Fabricación digital (con nuevas formas de diseño y manufacturas), R-FID (Rastreo Satelital, Logistic) en cadenas de producción, VAT (Drones con gran variedad de usos industriales) propiciando una mejora mediante la implantación de administración Inteligente, que sirve como estrategia para consolidar la sociedad tecnológica y del conocimiento en sistemas productivos mexicanos.

\section{Modelos Disruptivos, Robotica, LOT}

\begin{abstract}
Industry 4.0 in Mexico, intelligent management in productive systems A disruptive business model is that scheme that has changed according to the new consumer trends of the customers, turning a physical production industry into a form of electronic production, opening new opportunities for the industry in Mexico and becoming an element economic and social transformer that have allowed to change the rules of the game, through implementation of technological developments, which are creating an impact on the way of doing business, through technologies such as artificial intelligence (with machines capable of learning), Robotics (enhancing Automation), Internet of Things (with an interconnected world), Digital Manufacturing (with new forms of design and manufacturing), R-FID (Satellite Tracking, Logistic) in production chains, VAT (Drones with a variety of industrial uses ) fostering an improvement through the implementation of Intelligent Management, which serves as an strategy to consolidate the technological and knowledge society in Mexican productive systems.
\end{abstract}

Disruptive business model, Artificial intelligence, Digital Manufacturing

\footnotetext{
* Correspondencia del Autor (Correo electrónico: mpintor@ tesji.edu.mx)

$\dagger$ Investigador contribuyendo como primer autor.
} 


\section{Introducción}

Una industria desafiante nos espera, en los próximos años alcanzaremos los 50 millones de dispositivo conectados a internet, por menos es el panorama en México, de acuerdo a la revista INFO CANNEL, SEMANARIO PARA LA INDUSTRIAL TIC ABRIL 2019. (Arenas, 2019). Declara que más del $50 \%$ de las empresas a nivel mundial tiene planes de adoptar soluciones de Internet of things (IIOT), lo que traen consigo la incorporación de la Industria 4.0; administración inteligente en sistemas productivos.

\section{Industria 4.0 en México, la administración inteligente en sistemas productivos}

En el caso de la industria manufacturera podría alcanzar un nivel de digitalización del $70 \%$ de su producción, así obtener una reducción de costos de más del 400 millones de dólares, ya que dotará de inteligencia a los sistemas de producción por lo que se convierte en una tendencia de conexión y operación inteligente de máquinas, estos sistemas serán capaces de procesar pedidos en línea, ser surtidos al recolectar datos y transformarlos con la ayuda de la ingeniería de datos (analítica); la cual, busca tendencias mundiales en el consumo de productos, para dar una respuesta ágil a los consumidores. (Aguilar, 2013)

La revolución industrial debido a su costo beneficio, relacionado con la integración, la innovación y la automatización de los procesos. Contempla la tecnología 4.0 y la manufactura inteligente en la industria manufacturera, con procesos productivos totalmente integrados con resultados positivos en la eficiencia operativa y el desempeño organizacional, impactando a la visión y actuación empresarial.

Sin embargo hoy en día aun con la industria 4.0 y la manufactura inteligente, muchos empresarios prefieren esperar por el desconocimiento de los resultados que se obtienen con la tecnología aunada a que no hay información suficiente sobre su potencial que trae a las empresas. (Casapia, 2019)

El objetivo de este trabajo es indagar sobre el entorno de la Industria 4.0, las ventajas, desventajas, oportunidades y beneficios.
Difundir como estas pueden transformar las empresas productivas y sobre todo a las PYMES y concientizar a los empresarios sobre la importancia de trasladarse a la industria 4.0 para ser competitivos y sobrevivir en un mercado, globalizado dando oportunidad de internarnos a otros países, siendo proveedores globales.

\section{Aumenta 99.3\% consumo de datos móviles en México en primer trimestre del 2019.}

Según El Economista, en el 2019 México se ha considerado el primer país en consumo de datos a nivel mundial, lo que representa un reto para la industria de consumo y producción, en el Gráfico consumo de datos en México.

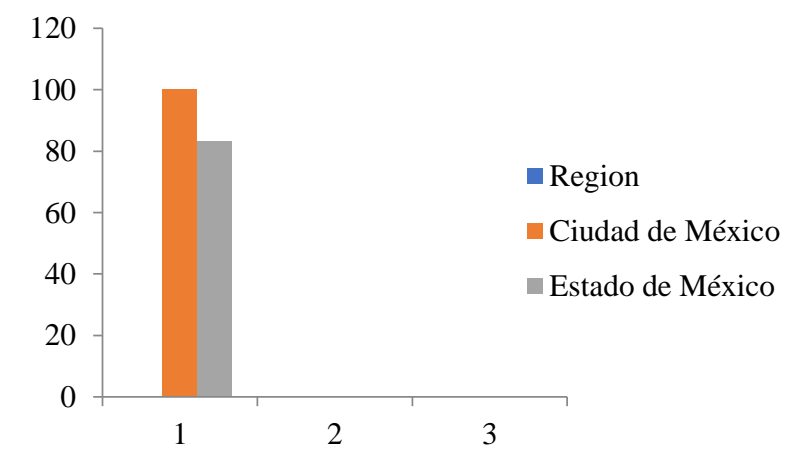

Grafico 1 Consumo de datos en México

Al primer trimestre de este año, señaló la consultora The Competitive Intelligence Unit, cada usuario móvil consumió en promedio 2,066 megabytes (MB) al mes, $99.3 \%$ más de lo reportado en el mismo periodo del año anterior, por lo tanto podemos predecir que tanto la tecnología como los dispositivos de conexión no serán suficientes para la nueva industria 4.0, de acuerdo con Gartner, al testear la tecnología, se puede sustituir en un lapso de tiempo aproximado de un año. (Monroy, 2019)

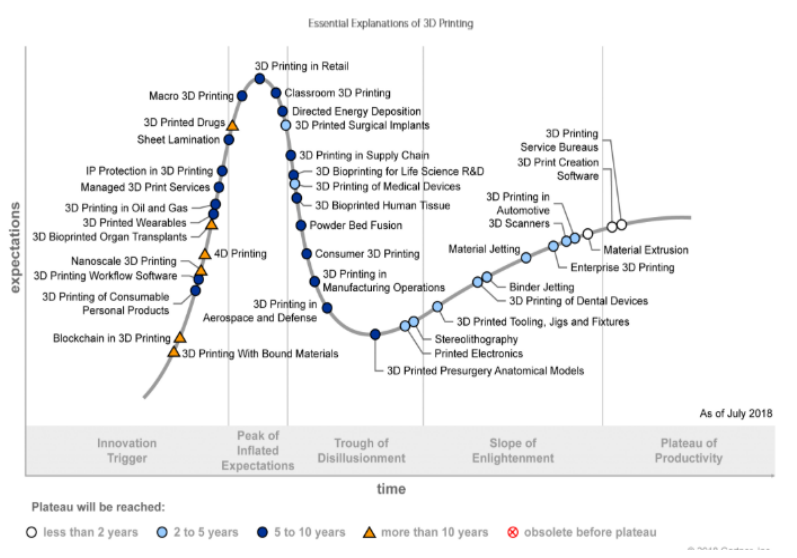

Figura 1 Gartner testeo y productos tecnológicos 2019 


\begin{tabular}{|l|r|}
\hline Consumo de datos & \% de consumo \\
\hline Redes sociales & $60 \%$ \\
\hline Comunicaciones & $20 \%$ \\
\hline Inteligencia artificial & $17 \%$ \\
\hline Ciencias de datos & $3 \%$ \\
\hline
\end{tabular}

Tabla 1 Consumo de datos en México

\section{Metodología a desarrollar}

La metodología es a partir de la mixta, llevándose a cabo entrevistas en 6 empresas de la región para medir la tecnología que se utiliza, se observó los procesos de las diferentes empresas, con los cuales nos arroja que las empresas de la región de Jilotepec se niegan a adoptar tecnología por el costo tan alto que se tiene que invertir, otro factor es la incertidumbre de la reacción que pueda ocasionar entre los colaboradores y la tercera es la falta de información y asesoría sobre los beneficios que tendrían a corto plazo.

Los estudios que se están realizando en los procesos para hacer la comparación con el proceso que haría con tecnología y hacer los cálculos para tener resultados que se puedan medir y darlos a conocer a los dueños de las empresa ayudarlos a tomar decisiones a que deben adoptar e implementar tecnología en sus procesos.

\section{Resultados}

Los resultados que se han obteniendo es participar en diversas conferencias: sobre el tema de industria 4.0, la administración inteligente en sistemas productivos (Corona, 2019), donde se aborda la importancia de los negocios disruptivos y como incorporar tecnológica como agente de cambio en las empresas productoras y prestadoras de servicios.

\section{Agradecimiento}

El presente proyecto de investigación es financiado por el Tecnológico de Estudios Superiores de Jilotepec, con la finalidad de difundir los proyectos de investigación en colaboración del Instituto Tecnológico de Estudios Superiores de Jalisco.

\section{Conclusiones}

Los principales beneficios que se obtienen con este proyecto de investigación de la industria 4.0 la administración inteligente en sistemas, y iniciar el proceso de transformación productivos, claridad los resultados obtenidos y las posibilidades de mejora.

\section{Referencias}

Aguilar, L. J. (2013). Big Data analsis de grades volumes de datos en organizaciones. México: Alfaomega.

Arenas, A. (2019). IIOT Permite la operacion inteligente de maquinas para la optimizar lols procesos de produccion. Info Channel, 24.

Casapia, R. (14 de Junio de 2019). Foro de Enseñanza y Tecnología Educativa 2019. Toluca México, Estado de Mexico, Toluca.

Corona, M. P. (23 de junio de 2019). Industria 4.0 en México, la administración inteligente en sistemas productivos. (C. Caica, Intérprete) Auditorio de vinculacion, Jocotitlan México, Estado de México, Mexico.

Monroy, I. E. (2019). Edge Computing analisis $\mathrm{y}$ aplicaciones . Edge Computing analisis y aplicaciones (pág. www.jaltec.com.mx). Guadalajara Jalisco: NA. 